\title{
A MODE III INTERFACE CRACK WITH SURFACE STRAIN GRADIENT ELASTICITY
}

\author{
XU WANG AND PETER SCHIAVONE \\ Communicated by Johannes Tausch
}

\begin{abstract}
We study the contribution of surface strain gradient elasticity to the anti-plane deformations of an elastically isotropic bimaterial containing a mode III interface crack. The surface strain gradient elasticity is incorporated using an enriched version of the continuum-based surface/interface model of Gurtin and Murdoch. We obtain a complete semi-analytic solution valid everywhere in the solid (including at the crack tips) by reducing the boundary value problem to two coupled hyper-singular integro-differential equations which are solved numerically using Chebyshev polynomials and a collocation method. Our solution demonstrates that the presence of surface strain gradient elasticity on the crack faces leads to bounded stresses at the crack tips.
\end{abstract}

1. Introduction. The continuum-based surface/interface model of Gurtin and Murdoch $[4, \mathbf{5}, \mathbf{6}]$ is based on the idea of a two-dimensional membrane bonded to the surface of a three-dimensional bulk substrate $[13, \mathbf{1 7}, \mathbf{1 8}]$. Recently, the Gurtin-Murdoch model was incorporated into the study of deformations of linearly elastic materials containing a crack with sharp tips $[\mathbf{1}, \mathbf{8}, \mathbf{9}, \mathbf{1 0}, \mathbf{1 1}, \mathbf{1 2}, \mathbf{2 2}, \mathbf{2 3}, \mathbf{2 4}, \mathbf{2 6}, \mathbf{2 7}]$. It was subsequently observed that the incorporation of the Gurtin-Murdoch model will reduce the classical square root singularity predicted by linear elastic fracture mechanics (LEFM) to only a weak logarithmic

2010 AMS Mathematics subject classification. Primary 45E99, 74B05.

Keywords and phrases. Surface strain gradient elasticity, Mode III interface crack, isotropic bimaterial, stress singularity, bounded stresses, complete solution, Green's function method, hyper-singular integro-differential equation.

This work is supported by the National Natural Science Foundation of China (grant No. 11272121) and through a Discovery Grant from the Natural Sciences and Engineering Research Council of Canada (grant No. RGPIN 155112). The second author is the corresponding author.

Received by the editors on March 6, 2015, and in revised form on October 12, 2016. 
singularity $[\mathbf{7}, \mathbf{2 1}]$. Most recently, [25] observed the interesting phenomenon that if surface strain gradient elasticity developed within the framework of Gurtin-Murdoch surface elasticity $[4,5,6]$ and strain gradient elasticity $[\mathbf{3}, \mathbf{1 5}, \mathbf{2 0}]$ is incorporated into the analysis of an isotropic and homogeneous solid containing a mode III crack, all stress components are bounded at the crack tips and the crack opening displacement assumes a cusp shape.

In this paper, we further explore the idea of surface strain gradient elasticity in [25] and consider the much more significant problem concerning anti-plane shear deformations of a bimaterial consisting of two perfectly bonded dissimilar isotropic elastic half-planes containing a crack along the bimaterial interface. Using the method of Green's functions, we reduce the original boundary value problem to a system of two coupled hyper-singular integro-differential equations which is solved numerically using a Chebyshev polynomial expansion and a collocation method $[\mathbf{2}, \mathbf{8}, \mathbf{1 1}, \mathbf{1 5}]$. This leads to a complete semianalytic solution valid everywhere in the domain of interest including at the crack tips. The analytical and numerical results obtained here clearly indicate that the incorporation of surface strain gradient effects leads to bounded stresses at the interface crack tips and a crack opening displacement which assumes a cusp-shaped profile at the crack tips. We note also that the stress component $\sigma_{31}$ continues to experience a discontinuity across the bimaterial interface as previously predicted in [11] in the case of the classical Gurtin-Murdoch surface model.

\section{The coupled bulk-surface elasticity.}

2.1. The bulk elasticity. In a fixed rectangular coordinate system $x_{i}, i=1,2,3$, let $u_{i}, \sigma_{i j}$ and $\varepsilon_{i j}$ be the displacement, stress and strain components, respectively, in an elastically isotropic bulk material. The equations of equilibrium, stress-strain laws and strain-displacement relations are

$$
\sigma_{i j, j}=0, \quad \sigma_{i j}=2 \mu \varepsilon_{i j}+\lambda \varepsilon_{k k} \delta_{i j}, \quad \varepsilon_{i j}=\frac{1}{2}\left(u_{i, j}+u_{j, i}\right),
$$

where $(i, j, k=1,2,3), \lambda$ and $\mu$ are the Lame constants of the bulk and $\delta_{i j}$ is the Kronecker delta.

In the case of anti-plane shear deformations, the two shear stress components $\sigma_{31}$ and $\sigma_{32}$ and out-of-plane displacement $w=u_{3}\left(x_{1}, x_{2}\right)$ 
can be expressed in terms of a single analytic function $h(z)$ of the complex variable $z=x_{1}+i x_{2}$ as

$$
\sigma_{32}+i \sigma_{31}=\mu h^{\prime}(z), \quad w=\operatorname{Im}\{h(z)\} .
$$

2.2. The surface strain gradient elasticity. When the interface/surface mechanics of Gurtin and Murdoch is incorporated into the model of deformation, the equilibrium condition on a crack surface can be described by the following equations (see $[4,5,6,16]$ for detailed derivations)

$$
\begin{array}{ll}
{\left[\sigma_{\alpha_{j}} n_{j} \underline{e}_{\alpha}\right]+\sigma_{\alpha \beta, \beta}^{s} \underline{e}_{\alpha}=0} & \text { tangential direction } \\
{\left[\sigma_{i j} n_{i} n_{j}\right]=\sigma_{\alpha \beta}^{s} \kappa_{\alpha, \beta}} & \text { normal direction, }
\end{array}
$$

where $\alpha, \beta=1,3 ; \underline{e}_{\alpha}$ are the basis vectors describing the surface, $n_{i}$ is the unit normal vector to the surface, $[*]$ denotes the jump of the corresponding quantity across the surface, $\sigma_{\alpha \beta}^{s}$ are the components of the surface total stress tensor and $\kappa_{a \beta}$ are the components of the surface curvature tensor. In addition, the surface Cauchy stresses $\tau_{\alpha \beta}^{s}$, the surface couple stresses $\mu_{\omega \alpha \beta}^{\sigma}, \omega=1,3$, and the surface total stresses $\sigma_{\alpha \beta}^{s}$ are related to the deformation-dependent surface energy $\Gamma$ by $[3,15,20,25]$

$$
\begin{aligned}
\tau_{\alpha \beta}^{s} & =\sigma_{0} \delta_{\alpha \beta}+\frac{\partial \Gamma}{\partial \varepsilon_{\alpha \beta}^{s}}, \\
\mu_{\omega \alpha \beta}^{s} & =\frac{\partial \Gamma}{\partial \varepsilon_{\alpha \beta, \omega}^{s}}, \\
\sigma_{\alpha \beta}^{s} & =\tau_{\alpha \beta}^{s}-\mu_{\omega \alpha \beta, \omega}^{s},
\end{aligned}
$$

where $\varepsilon_{\alpha \beta}^{s}$ are the components of the surface strain tensor and $\sigma_{0}$ is the surface tension.

In this paper, the surface energy $\Gamma$ will take the following form

$$
\Gamma=\frac{1}{2}\left(\lambda^{s}+\sigma_{0}\right)\left(\varepsilon_{\alpha \alpha}^{s}\right)^{2}+\left(\mu^{s}-\sigma_{0}\right) \varepsilon_{\alpha \beta}^{s} \varepsilon_{\alpha \beta}^{s}+\left(\mu^{s}-\sigma_{0}\right) l^{2} \varepsilon_{\alpha \beta, \omega}^{s} \varepsilon_{\alpha \beta, \omega}^{s},
$$

where $\lambda^{s}$ and $\mu^{s}$ are the two surface Lame parameters and $l(>0)$ is a characteristic length. As a result, it follows from equations (2.4) and (2.5) that the surface total stresses can be expressed in terms of surface strains and surface strain derivatives as (Wang and Zhou [25])

$$
\sigma_{\alpha \beta}^{s}=\sigma_{0} \delta_{\alpha \beta}+2\left(\mu^{s}-\sigma_{0}\right)\left(\varepsilon_{\alpha \beta}^{s}-l^{2} \Delta_{s} \varepsilon_{\alpha \beta}^{s}\right)+\left(\lambda^{s}+\sigma_{0}\right) \varepsilon_{\gamma \gamma}^{s} \delta_{\alpha \beta},
$$


where $\Delta_{s}$ is the surface Laplacian operator.

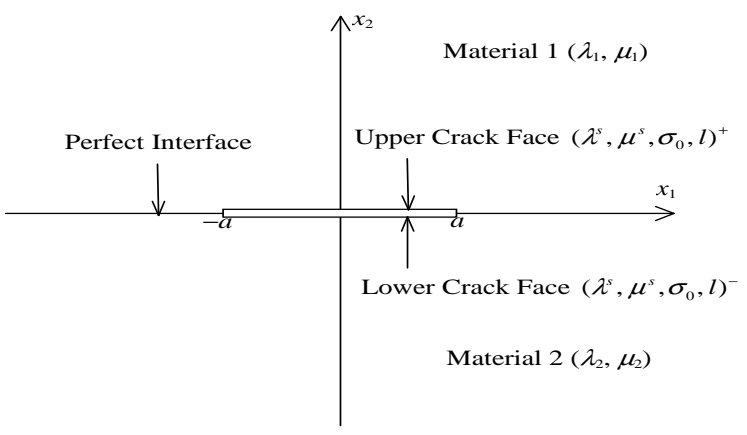

FIgURE 1. Schematic of the problem.

3. A mode III interface crack with strain gradient elasticity. As shown in Figure 1, we consider the anti-plane shear deformations of a solid consisting of two bonded dissimilar isotropic half-planes containing a finite interface crack, the cross section of which occupies the segment $[-a, a]$ of the real axis. The bimaterial is subjected to a uniform remote shear stress $\sigma_{32}^{\infty}$. Let the upper half-plane $\left(x_{2}>0\right.$, occupied by material "1") and the lower half-plane $\left(x_{2}<0\right.$, occupied by material "2") be designated the "+" and "-" sides of the crack, respectively. The bonded part of the bimaterial interface is perfectly bonded. In what follows, the subscripts 1 and 2 (or the superscripts (1) and (2)) are used to identify the respective quantities in the upper and lower half-planes.

From equation (2.3), the boundary conditions on the crack faces can be written as:

(3.1a) $\sigma_{13,1}^{s}+\left(\sigma_{23}\right)^{+}-\left(\sigma_{23}\right)^{-}=0$, on the upper crack face,

(3.1b) $\sigma_{13,1}^{s}+\left(\sigma_{23}\right)^{+}-\left(\sigma_{23}\right)^{-}=0, \quad$ on the lower crack face,

where, in the present crack problem, $\left(\sigma_{23}\right)^{-}$in equation (3.1a) and $\left(\sigma_{23}\right)^{+}$in equation $(3.1 \mathrm{~b})$ are zero. 
Under the assumption of a coherent interface $\left(\varepsilon_{\alpha \beta}^{s}=\varepsilon_{\alpha \beta}\right.$ and $\left.\varepsilon_{\alpha \beta, \omega}^{s}=\varepsilon_{\alpha \beta, \omega}\right)$, the surface stresses can be expressed as

$$
\sigma_{13}^{s}=\left(\mu^{s}-\sigma_{0}\right)\left(u_{3,1}-l^{2} u_{3,111}\right) .
$$

Insertion of equation (3.2) into equations (3.1a) and (3.1b) leads to:

$\left(\sigma_{23}\right)^{+}=-\left(\mu^{s}-\sigma_{0}\right)^{+}\left(u_{3,11}^{+}-l^{2} u_{3,1111}^{+}\right), \quad$ on the upper crack face,

$\left(\sigma_{23}\right)^{-}=+\left(\mu^{s}-\sigma_{0}\right)^{-}\left(u_{3,11}^{-}-l^{2} u_{3,1111}^{-}\right), \quad$ on the lower crack face,

where $\left(\mu^{s}-\sigma_{0}\right)^{+} \neq\left(\mu^{s}-\sigma_{0}\right)^{-}$. In writing equation (3.3), it has been assumed that $l^{+}=l^{-}=l$. The above is equivalent to

$$
\begin{aligned}
\left(\sigma_{23}\right)^{+}+\left(\sigma_{23}\right)^{-}= & -\left(\mu^{s}-\sigma_{0}\right)^{+}\left(u_{3,11}^{+}-l^{2} u_{3,1111}^{+}\right) \\
& +\left(\mu^{s}-\sigma_{0}\right)^{-}\left(u_{3,11}^{-}-l^{2} u_{3,1111}^{-}\right), \\
\left(\sigma_{23}\right)^{+}-\left(\sigma_{23}\right)^{-}= & -\left(\mu^{s}-\sigma_{0}\right)^{+}\left(u_{3,11}^{+}-l^{2} u_{3,1111}^{+}\right) \\
& -\left(\mu^{s}-\sigma_{0}\right)^{-}\left(u_{3,11}^{-}-l^{2} u_{3,1111}^{-}\right),
\end{aligned}
$$

The problem can now be formulated by considering a distribution of screw dislocations with density $b\left(x_{1}\right)$ and line forces with density $f\left(x_{1}\right)$ on the interface crack. Thus, the two analytic functions $h_{1}(z)$ and $h_{2}(z)$ in the upper and lower half-planes can be written as [19]

$$
\begin{aligned}
h_{1}(z)= & \frac{\mu_{2}}{\pi\left(\mu_{1}+\mu_{2}\right)} \int_{-a}^{a} b(\xi) \log (z-\xi) d \xi \\
& -\frac{i}{\pi\left(\mu_{1}+\mu_{2}\right)} \int_{-a}^{a} f(\xi) \log (z-\xi) d \xi+\frac{\sigma_{32}^{\infty}}{\mu_{1}} z, \\
h_{2}(z)= & \frac{\mu_{1}}{\pi\left(\mu_{1}+\mu_{2}\right)} \int_{-a}^{a} b(\xi) \log (z-\xi) d \xi \\
& -\frac{i}{\pi\left(\mu_{1}+\mu_{2}\right)} \int_{-a}^{a} f(\xi) \log (z-\xi) d \xi+\frac{\sigma_{32}^{\infty}}{\mu_{2}} z,
\end{aligned}
$$

where $b(\xi)$ and $f(\xi)$ are two unknown density functions which are assumed to be sufficiently smooth in the interval $(-a, a)$. The following limiting values can then be obtained

$$
\left[h_{1}^{\prime}\left(x_{1}\right)\right]^{+}=-\frac{\mu_{2} i}{\mu_{1}+\mu_{2}} b\left(x_{1}\right)+\frac{\mu_{2}}{\pi\left(\mu_{1}+\mu_{2}\right)} \int_{-a}^{a} \frac{b(\xi)}{x_{1}-\xi} d \xi
$$




$$
\begin{aligned}
& -\frac{f\left(x_{1}\right)}{\mu_{1}+\mu_{2}}-\frac{i}{\pi\left(\mu_{1}+\mu_{2}\right)} \int_{-a}^{a} \frac{f(\xi)}{x_{1}-\xi} d \xi+\frac{\sigma_{32}^{\infty}}{\mu_{1}}, \\
{\left[h_{2}^{\prime}\left(x_{1}\right)\right]^{-}=} & \frac{\mu_{1} i}{\mu_{1}+\mu_{2}} b\left(x_{1}\right)+\frac{\mu_{1}}{\pi\left(\mu_{1}+\mu_{2}\right)} \int_{-a}^{a} \frac{b(\xi)}{x_{1}-\xi} d \xi \\
& +\frac{f\left(x_{1}\right)}{\mu_{1}+\mu_{2}}-\frac{i}{\pi\left(\mu_{1}+\mu_{2}\right)} \int_{-a}^{a} \frac{f(\xi)}{x_{1}-\xi} d \xi+\frac{\sigma_{32}^{\infty}}{\mu_{2}},
\end{aligned}
$$

where $-a<x_{1}<a$ and the integrals are defined in the sense of Cauchy principal value. Here, the superscript "+" denotes the limiting value on approaching the interface crack from the upper half-plane, while the superscript "-" indicates that the limiting value is obtained by approaching the interface crack from the lower half-plane.

Using the expressions of $h_{1}(z)$ and $h_{2}(z)$ in equation (3.6), the satisfaction of the boundary conditions in equations (3.4) and (3.5) will yield the following hyper-singular integro-differential equations

$$
\begin{array}{r}
(3.9)-\frac{2 \mu_{1} \mu_{2}}{\pi} \int_{-a}^{a} \frac{b(\xi)}{\xi-x_{1}} d \xi+\left(\mu_{2}-\mu_{1}\right) f\left(x_{1}\right)+2 s_{32}^{\infty}\left(\mu_{1}+\mu_{2}\right) \\
=\left[\mu_{2}\left(\mu^{s}-\sigma_{0}\right)^{+}+\mu_{1}\left(\mu^{s}-\sigma_{0}\right)^{-}\right]\left[b^{\prime}\left(x_{1}\right)-l^{2} b^{\prime \prime \prime}\left(x_{1}\right)\right] \\
-\frac{1}{\pi}\left[\left(\mu^{2}-\sigma_{0}\right)^{+}-\left(\mu^{s}-\sigma_{0}\right)^{-}\right]\left[\int_{-a}^{a} \frac{f(\xi)}{\left(\xi-x_{1}\right)^{2}} d \xi-6 l^{2} \int_{-a}^{a} \frac{f(\xi)}{\left(\xi-x_{1}\right)^{4}} \delta \xi\right], \\
-a<x_{1}<a,
\end{array}
$$

$$
\begin{aligned}
& \left(\mu_{1}+\mu_{2}\right) f\left(x_{1}\right)=\frac{1}{\pi}\left[\left(\mu^{s}-\sigma_{0}\right)^{+}+\left(\mu^{s}-\sigma_{0}\right)^{-}\right] \\
& \times\left[\int_{-a}^{a} \frac{f(\xi)}{\left(\xi-x_{1}\right)^{2}} d \xi-6 l^{2} \int_{-a}^{a} \frac{f(\xi)}{\left(\xi-x_{1}\right)^{4}} d \xi\right] \\
& -\left[\mu_{2}\left(\mu^{s}-\sigma_{0}\right)^{+}-\mu_{1}\left(\mu^{s}-\sigma_{0}\right)^{-}\right]\left[b^{\prime}\left(x_{1}\right)-l^{2} b^{\prime \prime \prime}\left(x_{1}\right)\right], \\
& -a<x_{1}<a,
\end{aligned}
$$

where all integrals exist as Hadamard finite parts with the exception of the integral on the left-hand side of equation (3.9), which is defined in the sense of Cauchy principal value.

It is further deduced from equations (3.7) and (3.8) that

$$
\Delta w=w^{+}-w^{-}=-\int_{-a}^{x_{1}} b(\xi) d \xi, \quad-a<x_{1}<a,
$$




$$
\sigma_{32}^{+}-\sigma_{32}^{-}=-f\left(x_{1}\right), \quad-a<x_{1}<a .
$$

Thus, the single valuedness of the displacement and the balance of force for a contour surrounding the crack surface require that

$$
\int_{-a}^{a} b(\xi) d \xi=0, \quad \int_{-a}^{a} f(\xi) d \xi=0 .
$$

Following Paulino et al. [15] and Martin [14], to uniquely define the unknown density functions $b\left(x_{1}\right)$ and $f\left(x_{1}\right)$, we further impose the following smooth closure conditions:

$$
\begin{aligned}
& b(a)=b(-a)=0, \\
& f(a)=f(-a)=0 .
\end{aligned}
$$

Here we add that $b\left(x_{1}\right)$ and $f\left(x_{1}\right)$ are, respectively, odd and even functions of $x_{1}$.

4. Solution to the hyper-singular integro-differential equations. Set $x=x_{1} / a$ and $t=\xi / a$ in equations (3.9), (3.10) and (3.13). For convenience, we write $b(x)=b(a x)=b\left(x_{1}\right)$ and $f(x)=f(a x)=$ $f\left(x_{1}\right)$. As a result, equations (3.9) and (3.10) can be written in the following normalized form

$$
\begin{aligned}
-\frac{1}{\pi} \int_{-1}^{1} \frac{\hat{b}(t)}{t-x} d t & +K \hat{f}(x)+2=S_{1}^{+}\left[\hat{b}^{\prime}(x)-\hat{l}^{2} \hat{b}^{\prime \prime \prime}(x)\right] \\
- & \frac{S_{2}^{-}}{\pi}\left[\int_{-1}^{1} \frac{\hat{f}(t)}{(t-x)^{2}} d t-6 \hat{l}^{2} \int_{-1}^{1} \frac{\hat{f}(t)}{(t-x)^{4}} d t\right]
\end{aligned}
$$

$$
\hat{f}(x)=\frac{S_{2}^{+}}{\pi}\left[\int_{-1}^{1} \frac{\hat{f}(t)}{(t-x)^{2}} d t-6 \hat{l}^{2} \int_{-1}^{1} \frac{\hat{f}(t)}{(t-x)^{4}} d t\right]-S_{1}^{-}\left[\hat{b}^{\prime}(x)-\hat{l}^{2} \hat{b}^{\prime \prime \prime}(x)\right],
$$

where $-1<x<1$ and

$$
\begin{aligned}
\hat{b}(x) & =\frac{2 \mu_{1} \mu_{2} b(x)}{\left(\mu_{1}+\mu_{2}\right) \sigma_{32}^{\infty}}, \quad \hat{f}(x)=\frac{f(x)}{\sigma_{32}^{\infty}}, \\
K & =\frac{\mu_{2}-\mu_{1}}{\mu_{1}+\mu_{2}}, \quad \hat{l}=\frac{l}{a},
\end{aligned}
$$




$$
\begin{aligned}
& S_{1}^{+}=\frac{\mu_{2}\left(\mu^{s}-\sigma_{0}\right)^{+}+\mu_{1}\left(\mu^{s}-\sigma_{0}\right)^{-}}{2 a \mu_{1} \mu_{2}}, \\
& S_{1}^{-}=\frac{\mu_{2}\left(\mu^{s}-\sigma_{0}\right)^{+}-\mu_{1}\left(\mu^{s}-\sigma_{0}\right)^{-}}{2 a \mu_{1} \mu_{2}}, \\
& S_{2}^{+}=\frac{\left(\mu^{s}-\sigma_{0}\right)^{+}+\left(\mu^{s}-\sigma_{0}\right)^{-}}{a\left(\mu_{1}+\mu_{2}\right)}, \\
& S_{2}^{-}=\frac{\left(\mu^{s}-\sigma_{0}\right)^{+}-\left(\mu^{s}-\sigma_{0}\right)^{-}}{a\left(\mu_{1}+\mu_{2}\right)} .
\end{aligned}
$$

We observe from the above expression that the two functions $\hat{b}(x)$ and $\hat{f}(x)$ and the six parameters $K, S_{1}^{+}, S_{1}^{-}, S_{2}^{+}, S_{2}^{-}$and $\hat{l}$ are dimensionless. Further, we deduce from equation (4.2) that the necessary and sufficient condition for $\hat{f}(x)=0$ is $S_{1}^{-}=0$, or equivalently, $\mu_{2}\left(\mu^{s}-\sigma_{0}\right)^{+}=$ $\mu_{1}\left(\mu^{2}-\sigma_{0}\right)^{-}$. The discussion in Wang and Zhou [25] for a mode III crack in a homogeneous material indeed satisfies the condition $S_{1}^{-}=0$.

For convenience, equations (4.1) and (4.2) are further expressed in the following equivalent form:

$$
\begin{aligned}
-\frac{1}{\pi} \int_{-1}^{1} \frac{\hat{b}(t)}{t-x} d t-\frac{S_{1}^{+} S_{2}^{+}-S_{1}^{-} S_{2}^{-}}{S_{2}^{+}}\left[\hat{b}^{\prime}(x)-\hat{l}^{2} \hat{b}^{\prime \prime \prime}(x)\right] \\
+\left(K+\frac{S_{2}^{-}}{S_{2}^{+}}\right) \hat{f}(x)=-2,
\end{aligned}
$$

$$
\begin{aligned}
&- \frac{S_{1}^{-}}{\pi S_{1}^{+}} \int_{-1}^{1} \frac{\hat{b}(t)}{t-x} d t \\
&-\frac{S_{1}^{+} S_{2}^{+}-S_{1}^{-} S_{2}^{-}}{\pi S_{1}^{+}}\left[\int_{-1}^{1} \frac{\hat{f}(t)}{(t-x)^{2}} d t-6 \hat{l}^{2} \int_{-1}^{1} \frac{\hat{f}(t)}{(t-x)^{4}} d t\right] \\
&+\left(1+\frac{K S_{1}^{-}}{S_{1}^{+}}\right) \hat{f}(x)=-\frac{2 S_{1}^{-}}{S_{1}^{+}}
\end{aligned}
$$

When $\mu_{1}=\mu_{2}$ and $\left(\mu^{s}-\sigma_{0}\right)^{+}=\left(\mu^{s}-\sigma_{0}\right)^{-}$, we have $S_{1}^{+}=S_{2}^{+}=$ $\left(\mu^{s}-\sigma_{0}\right) /(a \mu)$ and $K=S_{1}^{-}=S_{2}^{-}=0$. In this case, equation (4.5) is automatically satisfied, and equation (4.4) reduces to the corresponding result in Wang and Zhou [25].

The two unknown functions $\hat{b}(x)$ and $\hat{f}(x)$ can be approximated by 
the following weighted polynomials

$$
\begin{aligned}
& \hat{b}(x) \approx \hat{b}_{N}(x)=\sqrt{1-x^{2}} \sum_{m=0}^{N} A_{m} U_{m}(x), \\
& \hat{f}(x) \approx \hat{f}_{N}(x)=\sqrt{1-x^{2}} \sum_{m=0}^{N} B_{m} U_{m}(x),
\end{aligned}
$$

where $U_{m}(x)$ represents the $m$ th Chebyshev polynomial of the second kind, and $A_{m}$ and $B_{m}$ are unknown coefficients to be determined using the collocation method.

It is clear that the smooth closure conditions in equations (3.14) and (3.15) have been satisfied by using equation (4.6). In addition, the satisfaction of the two conditions in equation (3.13) yields

$$
A_{0}=0, \quad B_{0}=0
$$

Thus, $\hat{b}(x)$ and $\hat{f}(x)$ can further be expressed as

$$
\begin{aligned}
& \hat{b}(x) \approx \hat{b}_{N}(x)=\sqrt{1-x^{2}} \sum_{m=1}^{N} A_{m} U_{m}(x), \\
& \hat{f}(x) \approx \hat{f}_{N}(x)=\sqrt{1-x^{2}} \sum_{m=1}^{N} B_{m} U_{m}(x) .
\end{aligned}
$$

By substituting equation (4.8) into equations (4.4) and (4.5), and making use of the following identities $[\mathbf{2}, \mathbf{1 5}]$

$$
\begin{aligned}
& \frac{1}{\pi} \int_{-1}^{1} \frac{U_{m}(t) \sqrt{1-t^{2}}}{t-x} d t=-T_{m+1}(x), \\
& \frac{1}{\pi} \int_{-1}^{1} \frac{U_{m}(t) \sqrt{1-t^{2}}}{(t-x)^{2}} d t=-(m+1) U_{m}(x), \\
& \frac{1}{\pi} \int_{-1}^{1} \frac{U_{m}(t) \sqrt{1-t^{2}}}{(t-x)^{3}} d t \\
& = \begin{cases}0 & m=0, \\
\frac{m(m+1) U_{m+1}(x)-(m+1)(m+2) U_{m-1}(x)}{4\left(1-x^{2}\right)} & m \geq 1,\end{cases} \\
& \frac{1}{\pi} \int_{-1}^{1} \frac{U_{m}(t) \sqrt{1-t^{2}}}{(t-x)^{4}} d t \\
& = \begin{cases}0 & m=0,1, \\
\frac{\left[\begin{array}{l}
-(m+2)(m+1)(m+3) U_{m-2}(x) \\
+2\left(m^{2}-1\right)(m+3) U_{m}(x)-m\left(m^{2}-1\right) U_{m+2}(x)
\end{array}\right]}{24\left(1-x^{2}\right)^{2}} & m \geq 2,\end{cases}
\end{aligned}
$$




$$
\begin{aligned}
\frac{d}{d x}\left[U_{m}(x) \sqrt{1-x^{2}}\right] & =-\frac{(m+1) T_{m+1}(x)}{\sqrt{1-x^{2}}} \\
\frac{d^{2}}{d x^{2}}\left[U_{m}(x) \sqrt{1-x^{2}}\right]= & -(m+1) \frac{d}{d x}\left[\frac{T_{m+1}(x)}{\sqrt{1-x^{2}}}\right] \\
= & \frac{(m+1)\left[m T_{m+2}(x)-(m+2) T_{m}(x)\right]}{2\left(1-x^{2}\right)^{3 / 2}} \\
\frac{d^{3}}{d x^{3}}\left[U_{m}(x) \sqrt{1-x^{2}}\right]= & \frac{m(m+1)(m+2) T_{m+1}(x)}{\left(1-x^{2}\right)^{3 / 2}} \\
& +\frac{3(m+1) x\left[m T_{m+2}(x)-(m+2) T_{m}(x)\right]}{2\left(1-x^{2}\right)^{5 / 2}}
\end{aligned}
$$

with $T_{m}(x)$ being the $m$ th Chebyshev polynomial of the first kind, we arrive at the following equations

$$
\begin{aligned}
& \left(1-x^{2}\right)^{5 / 2} \sum_{m=1}^{N} A_{m} T_{m+1}(x)+\frac{S_{1}^{+} S_{2}^{+}-S_{1}^{-} S_{2}^{-}}{S_{2}^{+}}\left(1-x^{2}\right)^{2} \\
& \quad \times \sum_{m=1}^{N} A_{m}(m+1) T_{m+1}(x)+\frac{\hat{l}^{2}\left(S_{1}^{+} S_{2}^{+}-S_{1}^{-} S_{2}^{-}\right)}{S_{2}^{+}} \\
& \times \sum_{m=1}^{N} A_{m}(m+1)\left[m(m+2)\left(1-x^{2}\right) T_{m+1}(x)\right. \\
& \left.\quad+\frac{3 x\left[m T_{m+2}(x)-(m+2) T_{m}(x)\right]}{2}\right] \\
& +\left(K+\frac{S_{2}^{-}}{S_{2}^{+}}\right)\left(1-x^{2}\right)^{3} \sum_{m=1}^{N} B_{m} U_{m}(x)=-2\left(1-x^{2}\right)^{5 / 2},
\end{aligned}
$$

$$
\begin{aligned}
& \text { 12) } \begin{aligned}
\frac{S_{1}^{-}}{S_{1}^{+}}\left(1-x^{2}\right)^{2} \sum_{m=1}^{N} A_{m} T_{m+1}(x)+\frac{S_{1}^{+} S_{2}^{+}-S_{1}^{-} S_{2}^{-}}{S_{1}^{+}}\left(1-x^{2}\right)^{2} \\
\times \sum_{m=1}^{N} B_{m}(m+1) U_{m}(x)+\frac{\hat{l}^{2}\left(S_{1}^{+} S_{2}^{+}-S_{1}^{-} S_{2}^{-}\right)}{4 S_{1}^{+}} \\
\times \sum_{m=2}^{N} B_{m}\left[\begin{array}{l}
-(m+1)(m+2)(m+3) U_{m-2}(x) \\
+2\left(m^{2}-1\right)(m+3) U_{m}(x)-m\left(m^{2}-1\right) U_{m+2}(x)
\end{array}\right]
\end{aligned}
\end{aligned}
$$




$$
+\left(1+\frac{K S_{1}^{-}}{S_{1}^{+}}\right)\left(1-x^{2}\right)^{5 / 2} \sum_{m=1}^{N} B_{m} U_{m}(x)=-\frac{2 S_{1}^{-}}{S_{1}^{+}}\left(1-x^{2}\right)^{2} .
$$

Remark 4.1. The authors note an error in the coefficient appearing in equation (67) in Chan et al. [2].

If we select the collocation points given by $x=-\cos (i \pi / N)$ for $i=1,2, \ldots, N$, equations (4.11) and (4.12) further reduce to the following algebraic equations

$$
\begin{array}{r}
\sum_{m=1}^{N}(-1)^{m+1}\left[1-\left(\cos \left(\frac{i \pi}{N}\right)\right)^{2}\right]^{5 / 2} \\
\cos \left(\frac{(m+1) i \pi}{N}\right) A_{m}+\frac{S_{1}^{+} S_{2}^{+}-S_{1}^{-} S_{2}^{-}}{S_{2}^{+}}
\end{array}
$$$$
\times \sum_{m=1}^{N}(-1)^{m+1}(m+1)\left[1-\left(\cos \left(\frac{i \pi}{N}\right)\right)^{2}\right]^{2} \cos \left(\frac{(m+1) i \pi}{N}\right) A_{m}
$$

$$
\begin{gathered}
+\frac{S_{1}^{+} S_{2}^{+}-S_{1}^{-} S_{2}^{-}}{S_{2}^{+}} \hat{l}^{2} \\
\times \sum_{m=1}^{N}(-1)^{m+1}(m+1)\left[\begin{array}{l}
m(m+2)\left[1-\left(\cos \left(\frac{i \pi}{N}\right)\right)^{2}\right] \cos \left(\frac{(m+1) i \pi}{N}\right) \\
+\frac{3}{2} \cos \left(\frac{i \pi}{N}\right)\left[m \cos \left(\frac{(m+2) i \pi}{N}\right)-(m+2) \cos \left(\frac{m i \pi}{N}\right)\right]
\end{array}\right] A_{m} \\
+\left(K+\frac{S_{2}^{-}}{S_{2}^{+}}\right) \sum_{m=1}^{N}(-1)^{m}\left[1-\left(\cos \left(\frac{i \pi}{N}\right)\right)^{2}\right]^{3} \frac{\sin \left(\frac{(m+1) i \pi}{N}\right)}{\sin (i \pi / N)} B_{m} \\
=-2\left[1-\left(\cos \left(\frac{i \pi}{N}\right)\right)^{2}\right]^{5 / 2}, i=1,2, \ldots, N-1, \\
\sum_{m=1}^{N}(m+1) A_{m}=0,
\end{gathered}
$$

$$
\frac{S_{1}^{-}}{S_{1}^{+}} \sum_{m=1}^{N}(-1)^{m+1}\left[1-\left(\cos \left(\frac{i \pi}{N}\right)\right)^{2}\right]^{2} \cos \left(\frac{(m+1) i \pi}{N}\right) A_{m}
$$




$$
\begin{gathered}
+\frac{S_{1}^{+} S_{2}^{+}-S_{1}^{-} S_{2}^{-}}{S_{1}^{+}} \sum_{m=1}^{N}(-1)^{m}(m+1)\left[1-\left(\cos \left(\frac{i \pi}{N}\right)\right)^{2}\right]^{2} \\
\times \frac{\sin \left(\frac{(m+1) i \pi}{N}\right)}{\sin (i \pi / N)} B_{m}+\frac{\hat{l}^{2}\left(S_{1}^{+} S_{2}^{+}-S_{1}^{-} S_{2}^{-}\right)}{4 S_{1}^{+}} \\
\times \sum_{m=1}^{N}(-1)^{m}\left[\begin{array}{l}
\left.-(m+1)(m+2)(m+3) \frac{\sin \left(\frac{(m-1) i \pi}{N}\right)}{N}\right) \\
\left.+2\left(m^{2}-1\right)(m+3) \frac{\sin \left(\frac{(m+1) i \pi}{N}\right)}{\sin (i \pi / N)}-m\left(m^{2}-1\right) \frac{\sin \left(\frac{(m+3) i \pi}{N}\right)}{\sin (i \pi / N)}\right]
\end{array}\right] B_{m} \\
+\left(1+\frac{K S_{1}^{-}}{S_{1}^{+}}\right) \sum_{m=1}^{N}(-1)^{m}\left[1-\left(\cos \left(\frac{i \pi}{N}\right)\right)^{2}\right]^{5 / 2} \frac{\sin \left(\frac{(m+1) i \pi}{N}\right)}{\sin (i \pi / N)} B_{m} \\
=-\frac{2 S_{1}^{-}}{S_{1}^{+}}\left[1-\left(\cos \left(\frac{i \pi}{N}\right)\right)^{2}\right]^{2},
\end{gathered}
$$

If we take $i=N$ in equation (4.14), the two sides of this equation will be identically zero. In carrying out specific calculations, we take $N$ to be an even number. Due to symmetry, we further impose that $B_{m} \equiv 0$ for odd $m$. As a result, it is sufficient to take $i=1,2, \ldots, N / 2$ in equation (4.14). The $(N+N / 2)$ unknowns $A_{m}(m=1,2, \ldots, N)$ and $B_{2 m}(m=1,2, \ldots, N / 2)$ can then be uniquely determined by solving the $(N+N / 2)$ independent equations in (4.13) and (4.14).

5. The stress field. The stress field in the upper half-plane is determined by

$\sigma_{32}^{(1)}+i \sigma_{31}^{(1)}=\frac{\mu_{1} \mu_{2}}{\pi\left(\mu_{1}+\mu_{2}\right)} \int_{-1}^{a} \frac{b(\xi)}{z-\xi} d \xi-\frac{i \mu_{1}}{i\left(\mu_{1}+\mu_{2}\right)} \int_{-a}^{a} \frac{f(\xi)}{z-\xi} d \xi+\sigma_{32}^{\infty}$,

and the stress field in the lower half-plane is given by

$\sigma_{32}^{(2)}+i \sigma_{31}^{(2)}=\frac{\mu_{1} \mu_{2}}{\pi\left(\mu_{1}+\mu_{2}\right)} \int_{-a}^{a} \frac{b(\xi)}{z-\xi} d \xi-\frac{i \mu_{2}}{\pi\left(\mu_{1}+\mu_{2}\right)} \int_{-a}^{a} \frac{f(\xi)}{z-\xi} d \xi+\sigma_{32}^{\infty}$.

In view of the fact that $b(a)=b(-a)=f(a)=f(-a)=0$ in equations (3.14) and (3.15), the stresses are bounded at the crack tips. 


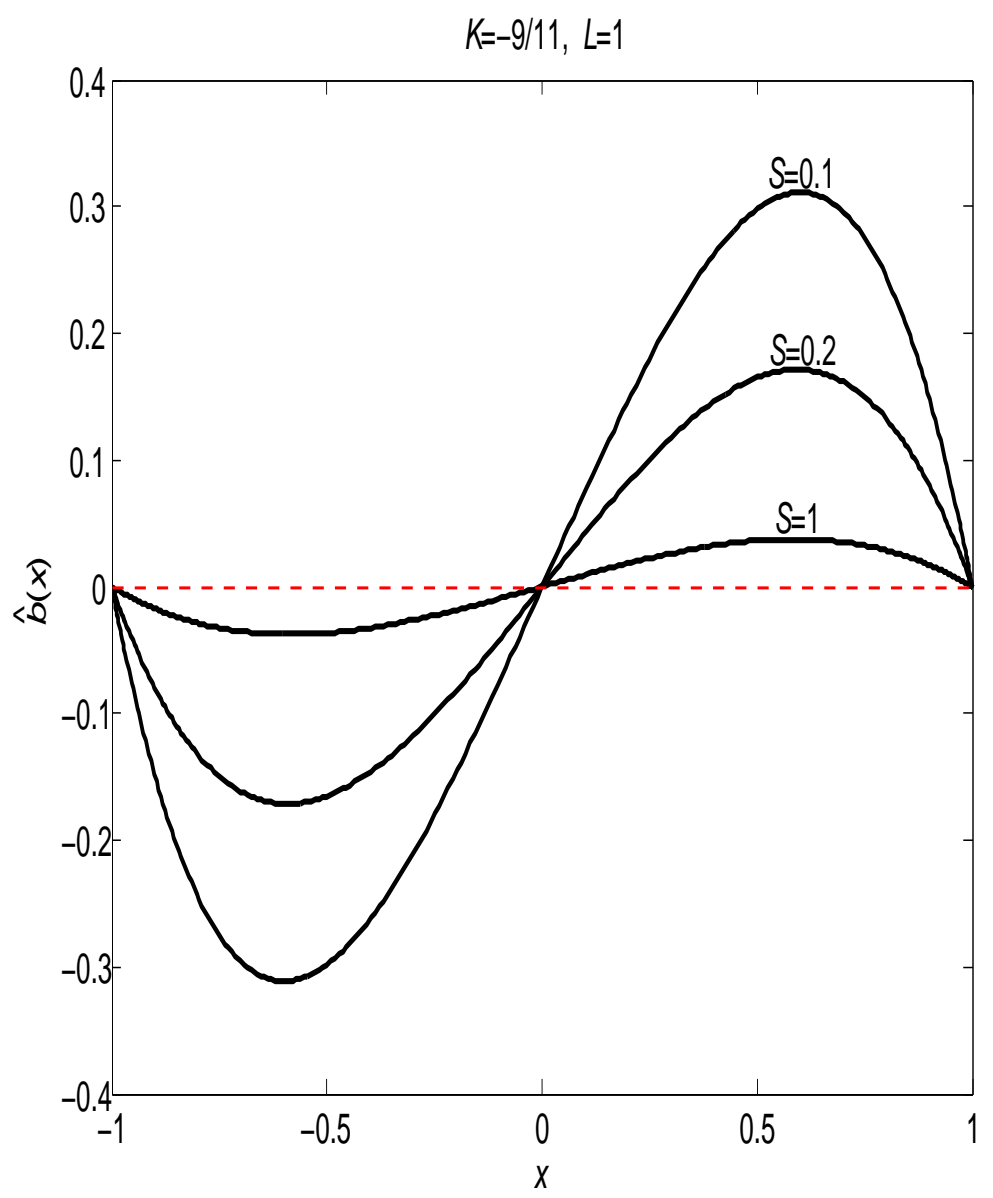

FiguRE 2. Variation of $\hat{b}(x)$ for three different values of $S=0.1,0.2,1$ with $K=-9 / 11$ and $L=1$.

In addition, by using the following formula [15]

(5.3) $\frac{1}{\pi} \int_{-1}^{1} \frac{\sqrt{1-t^{2}} U_{m}(t)}{t-x} d t=-\left(x-\frac{|x|}{x} \sqrt{x^{2}-1}\right)^{m+1}, \quad|x|>1$,

the stresses are distributed along the real axis outside the crack as 


$$
\begin{aligned}
\sigma_{32}^{(1)} & =\sigma_{32}^{(2)}=\frac{\sigma_{32}^{\infty}}{2 \pi} \int_{-1}^{1} \frac{\hat{b}(t)}{x-t} d t+\sigma_{32}^{\infty} \\
& =\frac{\sigma_{32}^{\infty}}{2} \sum_{m=1}^{N} A_{m}\left(x-\frac{|x|}{x} \sqrt{x^{2}-1}\right)^{m+1}+\sigma_{32}^{\infty}, \\
\sigma_{31}^{(1)} & =\frac{\sigma_{32}^{\infty}(1-K)}{2 \pi} \int_{-1}^{1} \frac{\hat{f}(t)}{t-x} d t \\
& =\frac{\sigma_{32}^{\infty}(K-1)}{2} \sum_{m=1}^{N} B_{m}\left(x-\frac{|x|}{x} \sqrt{x^{2}-1}\right)^{m+1}, \\
\sigma_{31}^{(2)} & =\frac{\sigma_{32}^{\infty}(1+K)}{2 \pi} \int_{-1}^{1} \frac{\hat{f}(t)}{t-x} d t=\frac{1+K}{1-K} \sigma_{31}^{(1)}, \quad|x|>1 .
\end{aligned}
$$

The stresses are distributed on the crack faces as

$$
\begin{aligned}
\sigma_{32}^{(1)}= & \frac{\sigma_{32}^{\infty}}{2} \sum_{m=1}^{N} A_{m} T_{m+1}(x)+\frac{\sigma_{32}^{\infty}(K-1)}{2} \sqrt{1-x^{2}} \sum_{m=1}^{N} B_{m} U_{m}(x)+\sigma_{32}^{\infty} \\
\sigma_{31}^{(1)}= & -\frac{\sigma_{32}^{\infty}}{2} \sqrt{1-x^{2}} \sum_{m=1}^{N} A_{m} U_{m}(x) \\
& +\frac{\sigma_{32}^{\infty}(K-1)}{2} \sum_{m=1}^{N} B_{m} T_{m+1}(x), \\
\sigma_{32}^{(2)}= & \frac{\sigma_{32}^{\infty}}{2} \sum_{m=1}^{N} A_{m} T_{m+1}(x)+\frac{\sigma_{32}^{\infty}(1+K)}{2} \sqrt{1-x^{2}} \sum_{m=1}^{N} B_{m} U_{m}(x)+\sigma_{32}^{\infty} \\
\sigma_{31}^{(2)}= & \frac{\sigma_{32}^{\infty}}{2} \sqrt{1-x^{2}} \sum_{m=1}^{N} A_{m} U_{m}(x) \\
& -\frac{\sigma_{32}^{\infty}(1+K)}{2} \sum_{m=1}^{N} B_{m} T_{m+1}(x), \quad-1<x<1 .
\end{aligned}
$$

It is observed from equations (5.4) and (5.5) that the stress components $\sigma_{32}^{(1)}, \sigma_{32}^{(2)}, \sigma_{31}^{(1)}$ and $\sigma_{31}^{(2)}$ on the bimaterial interface are all continuous 


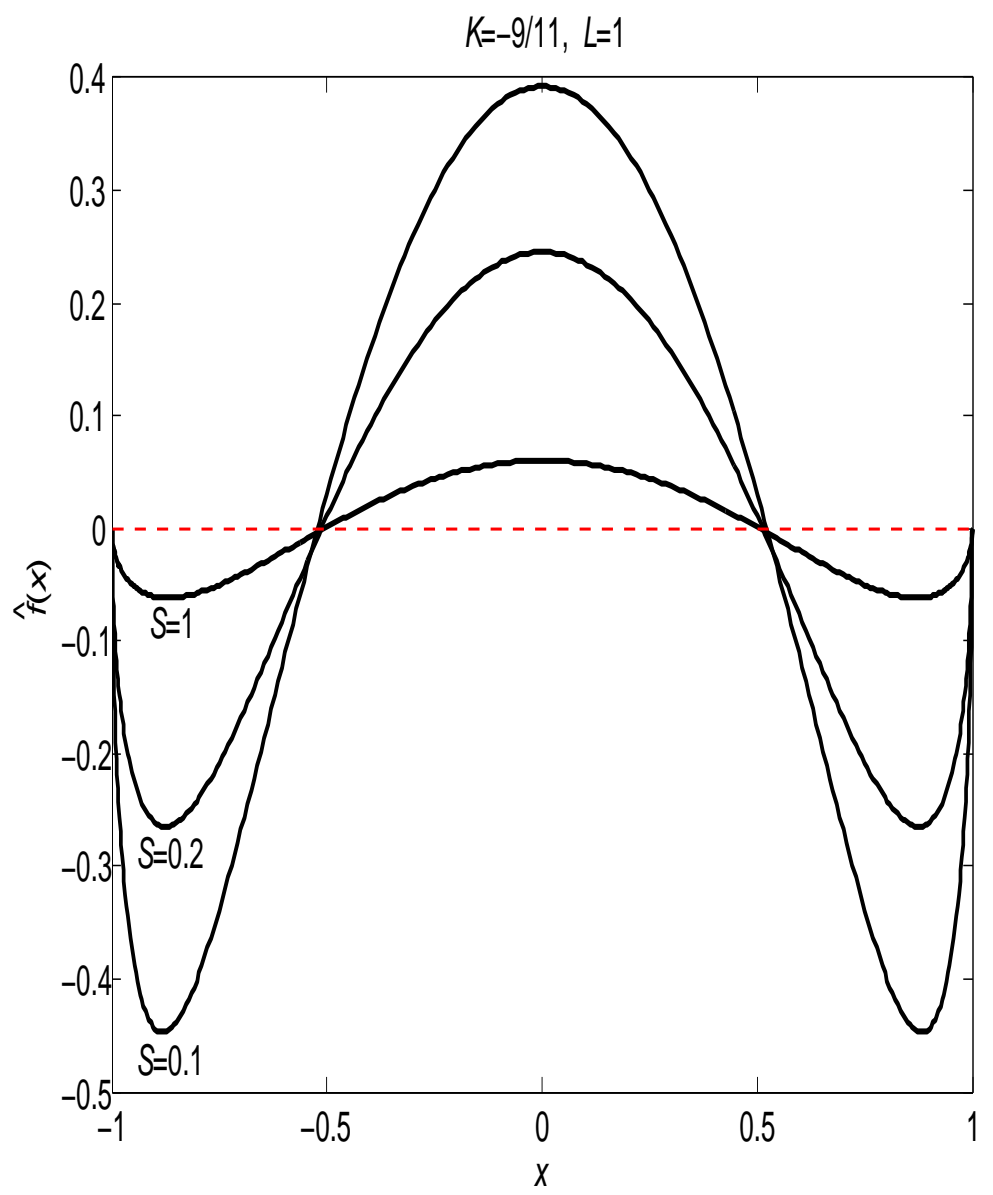

FiguRE 3. Variation of $\hat{f}(x)$ for three different values of $S=0.1,0.2,1$ with $K=-9 / 11$ and $L=1$.

across the crack tips. The present model also predicts that the stress component $\sigma_{31}$ is discontinuous across the bimaterial interface, i.e., $\sigma_{31}^{(1)} \neq \sigma_{31}^{(2)}$ for $|x|>1$, as in Kim et al. [11]. This discontinuity in stress stems from the fact that $f(x) \neq 0$ when $S_{1}^{-} \neq 0$. 


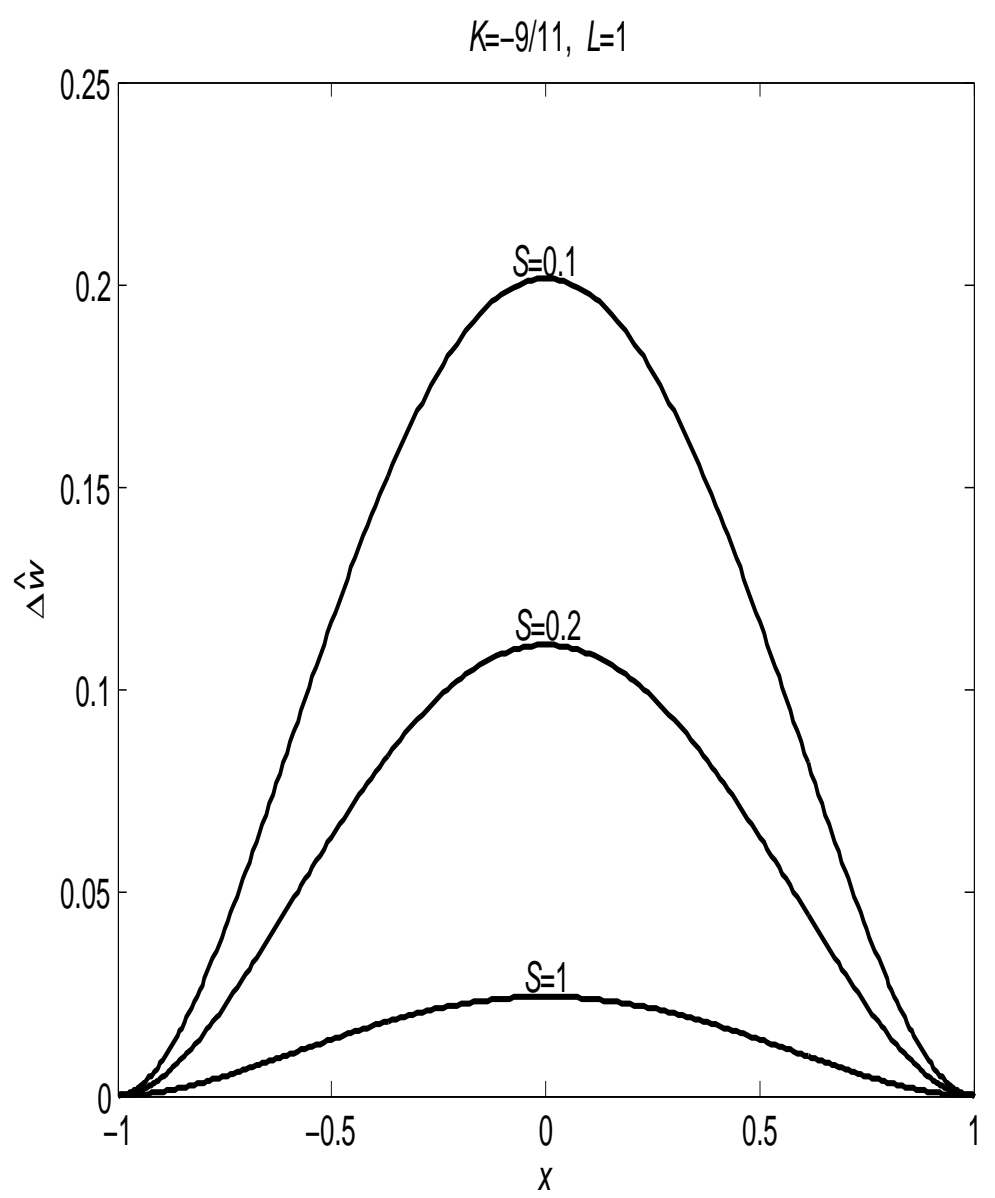

FiguRE 4. Variation of $\Delta \widehat{w}$ for three different values of $S=0.1,0.2,1$ with $K=-9 / 11$ and $L=1$.

6. Results and discussions. The crack opening displacement can be evaluated as (6.1)

$\Delta w=-a \int_{-1}^{x} b(t) d t=\frac{a \sigma_{32}^{\infty}\left(\mu_{1}+\mu_{2}\right)}{4 \mu_{1} \mu_{2}} \sum_{m=1}^{N} A_{m}\left[\frac{\sin m \theta}{m}-\frac{\sin (m+2) \theta}{m+2}\right], \cos \theta=x$. 


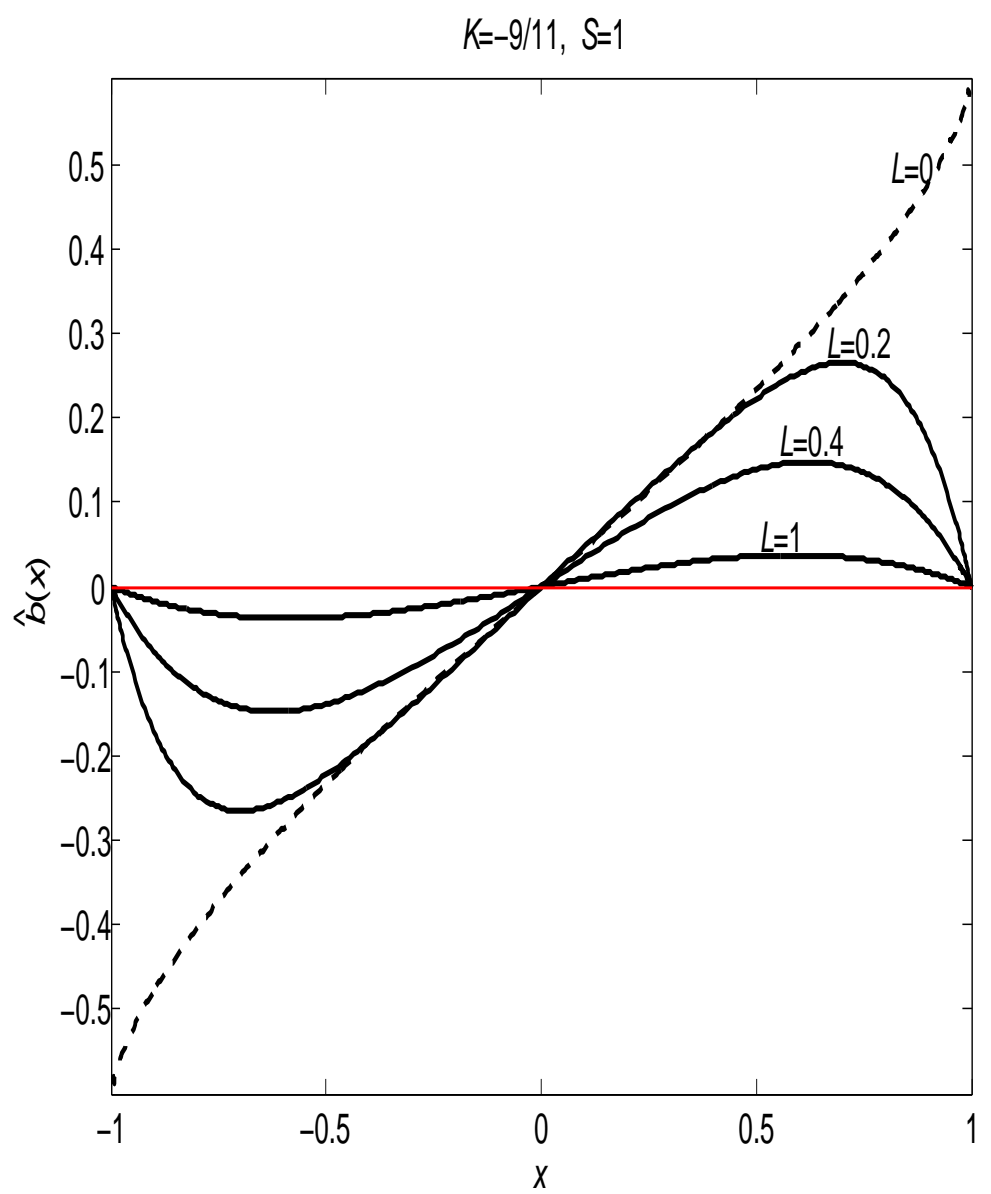

FIGURE 5. Variation of $\hat{b}(x)$ for four different values of $L=0,0.2,0.4,1$ with $K=-9 / 11$ and $S=1$.

In the following calculations, it is assumed that $\left(\mu^{s}-\sigma_{0}\right)^{+}=\left(\mu^{s}-\sigma_{0}\right)^{-}$. In this case, we have

$$
S_{2}^{+}=\frac{2\left(\mu^{s}-\sigma_{0}\right)}{a\left(\mu_{1}+\mu_{2}\right)}, \quad S_{1}^{+}=\frac{S_{2}^{+}}{1-K^{2}},
$$




$$
S_{1}^{-}=K S_{1}^{+}=\frac{K S_{2}^{+}}{1-K^{2}}, \quad S_{2}^{-}=0 .
$$

It is then observed from equations (4.4) and (4.5) that the three dimensionless functions $\widehat{b}(x), \hat{f}(x)$ and $\Delta \widehat{w}=\left(2 \mu_{1} \mu_{2} \Delta w\right) /\left(a \sigma_{32}^{\infty}\left(\mu_{1}+\right.\right.$ $\left.\mu_{2}\right)$ ) depend only on the three dimensionless parameters $K, S=s_{2}^{+}$and $L=\hat{l}$. In passing, we note that our numerical results in the case $\mu_{1}=\mu_{2}$ indeed recover the results in [25] for a crack in a homogeneous material. In the following numerical examples, we take the value $N=300$.

Illustrated in Figures $2-4$ are the variations of $\widehat{b}(x), \widehat{f}(x)$ and $\Delta \widehat{w}$ for the three values of $S=0.1,0.2,1$ with $K=-9 / 11$ and $L=1$. $K=-9 / 11$ is equivalent to $\mu_{1}=10 \mu_{2}$. The three smooth curves of $\widehat{b}(x)$ in Figure 2 satisfy $\widehat{b}(1)=\widehat{b}(-1)=0$ while the three smooth curves of $\widehat{f}(x)$ in Figure 3 satisfy $\widehat{f}(1)=\widehat{f}(-1)=0$. It is noted that $\Delta \widehat{w}$ in Figure 4 takes a cusp shape at $x= \pm 1$, as required for bounded stresses and strains at the crack tips. The function $\widehat{b}(x)$ in Figure 2 is an odd function of $x$, whilst $\widehat{f}(x)$ and $\Delta \widehat{w}$ in Figures 3 and 4 , respectively, are even functions of $x$. All of these observations are in agreement with our theoretical predictions. An increase in $S$ will suppress the magnitudes of $\widehat{b}(x), \widehat{f}(x)$ and $\Delta \widehat{w}$. In addition, the classical results corresponding to $\widehat{b}(x)=(2 x) / \sqrt{1-x^{2}}$ and $\Delta \widehat{w}=2 \sqrt{1-x^{2}}$ in LEFM can be obtained by setting $S$ and $L$ close to zero, which validates our results.

We show in Figures $5-7$ the variations of $\widehat{b}(x), \widehat{f}(x)$ and $\Delta \widehat{w}$ for four different values of $L=0,0.2,0.4,1$ with $K=-9 / 11$ and $S=1$. The result for the case $L=0$ is obtained using a very small yet nonzero value of $L$ (say, $L=0.00001$ ). The three smooth curves of the function $\widehat{b}(x)$ for $L \neq 0$ in Figure 5 satisfy $\widehat{b}(1)=\hat{b}(1)=0$, whereas that for $L=0$ in Figure 5 satisfies $\widehat{b}(1)=-\widehat{b}(-1)=0.59 \neq 0$; meanwhile, the three smooth curves of $\widehat{f}(x)$ for $L \neq 0$ in Figure 6 satisfy $\hat{f}(1)=\widehat{f}(-1)=0$, whereas the corresponding graph in the case of $L=0$ (Figure 6) is unbounded at $x= \pm 1$. The term $\Delta \widehat{w}$ for $L \neq 0$ in Figure 7 takes a cusp shape at $x= \pm 1$, the crack opening angle for $L=0$ in Figure 7 is strictly positive but less than $\pi / 2$. An increase in $L$ will again suppress the magnitudes of $\widehat{b}(x), \widehat{f}(x)$ and $\Delta \widehat{w}$. The nonzero finite values of $\widehat{b}( \pm 1)$ in Figure 5 and the infinite values of $\widehat{f}( \pm 1)$ for $L=0$ in Figure 6 imply that the stresses exhibit both the weak logarithmic and the strong square root singularities at the crack tips when the strain 


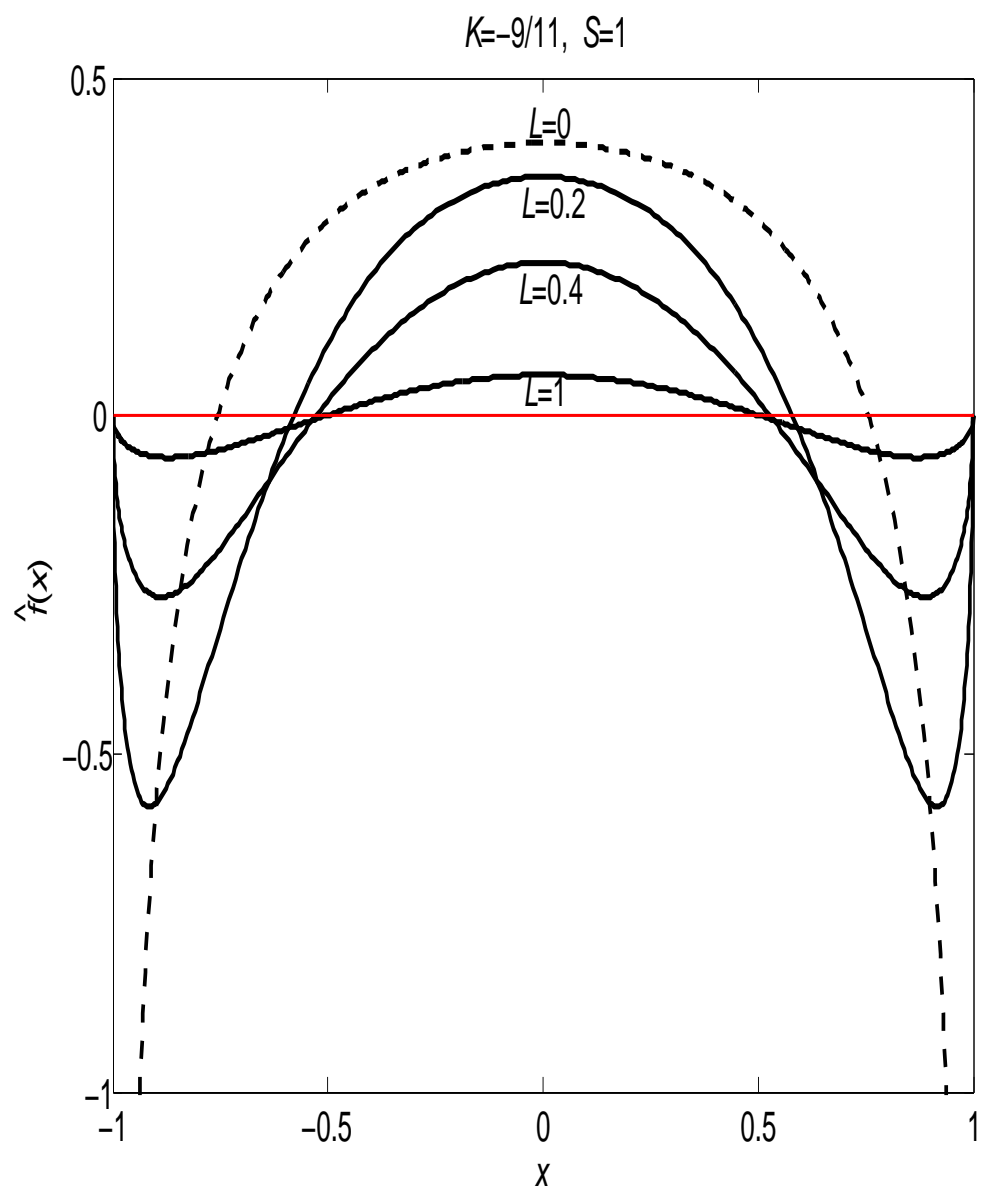

FiguRE 6. Variation of $\widehat{f}(x)$ for four different values of $L=0,0.2,0.4,1$ with $K=-9 / 11$ and $S=1$.

gradient effects of the crack faces are ignored [22].

Figure 8 shows the variation of $\widehat{f}(x)$ for different values of the mismatch parameter $K$ with $S=1$ and $L=0.2$. Apparently, a nonzero value of $K$ will induce a nontrivial distribution of $\hat{f}(x)$. We note that 


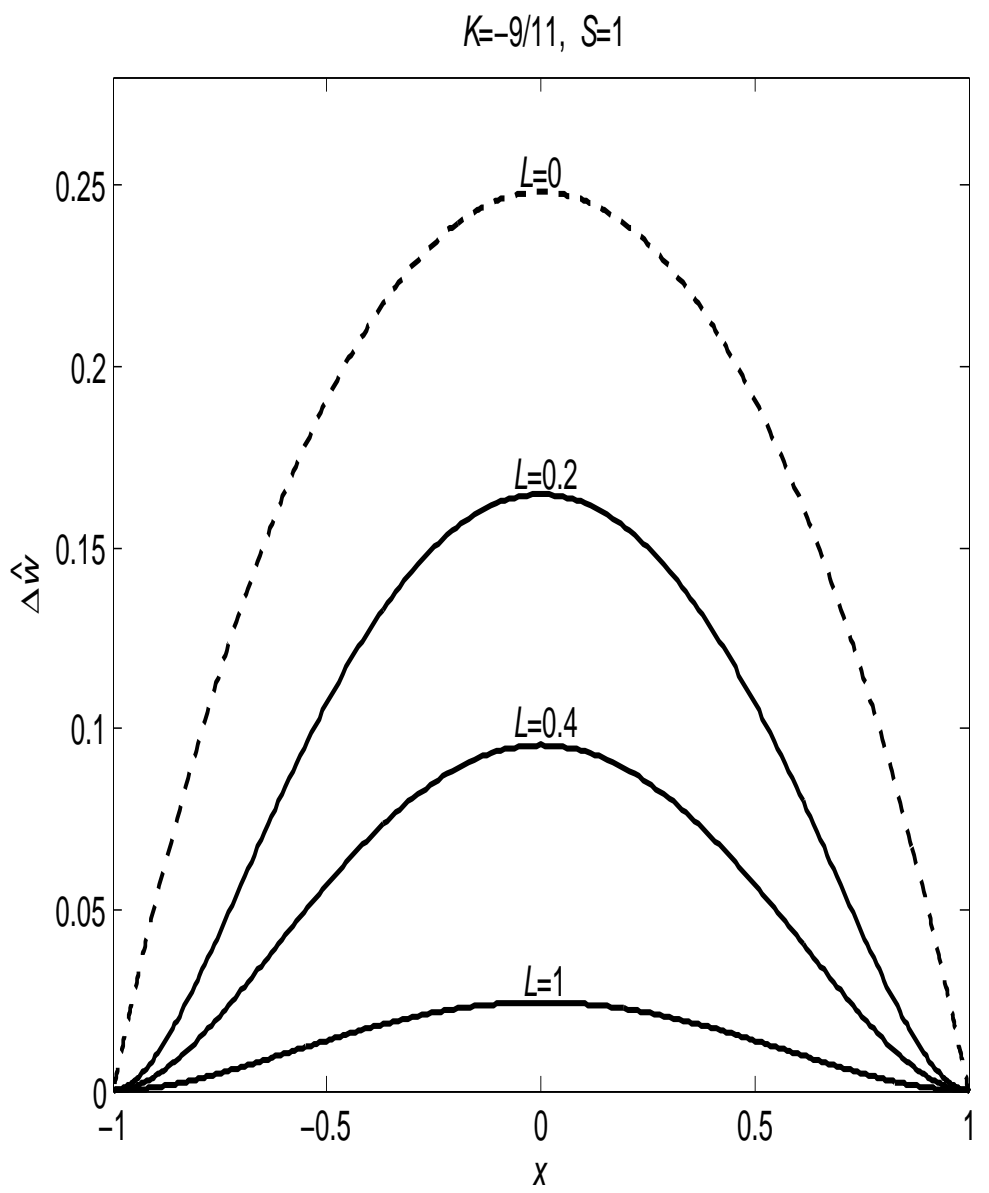

FIGURE 7. Variation of $\Delta \widehat{w}$ for four different values of $L=0,0.2,0.4,1$ with $K=-9 / 11$ and $S=1$.

$\widehat{f}(x) \equiv 0$ when $K=0$ (i.e., the crack lies in a homogeneous material).

We show in Figure 9 the continuous distributions of the stress components along the bimaterial interface with $K=-9 / 11, S=1$ and $L=0.2$. All the stress components are bounded at the crack tips. It is further observed from Figure 9 that: 


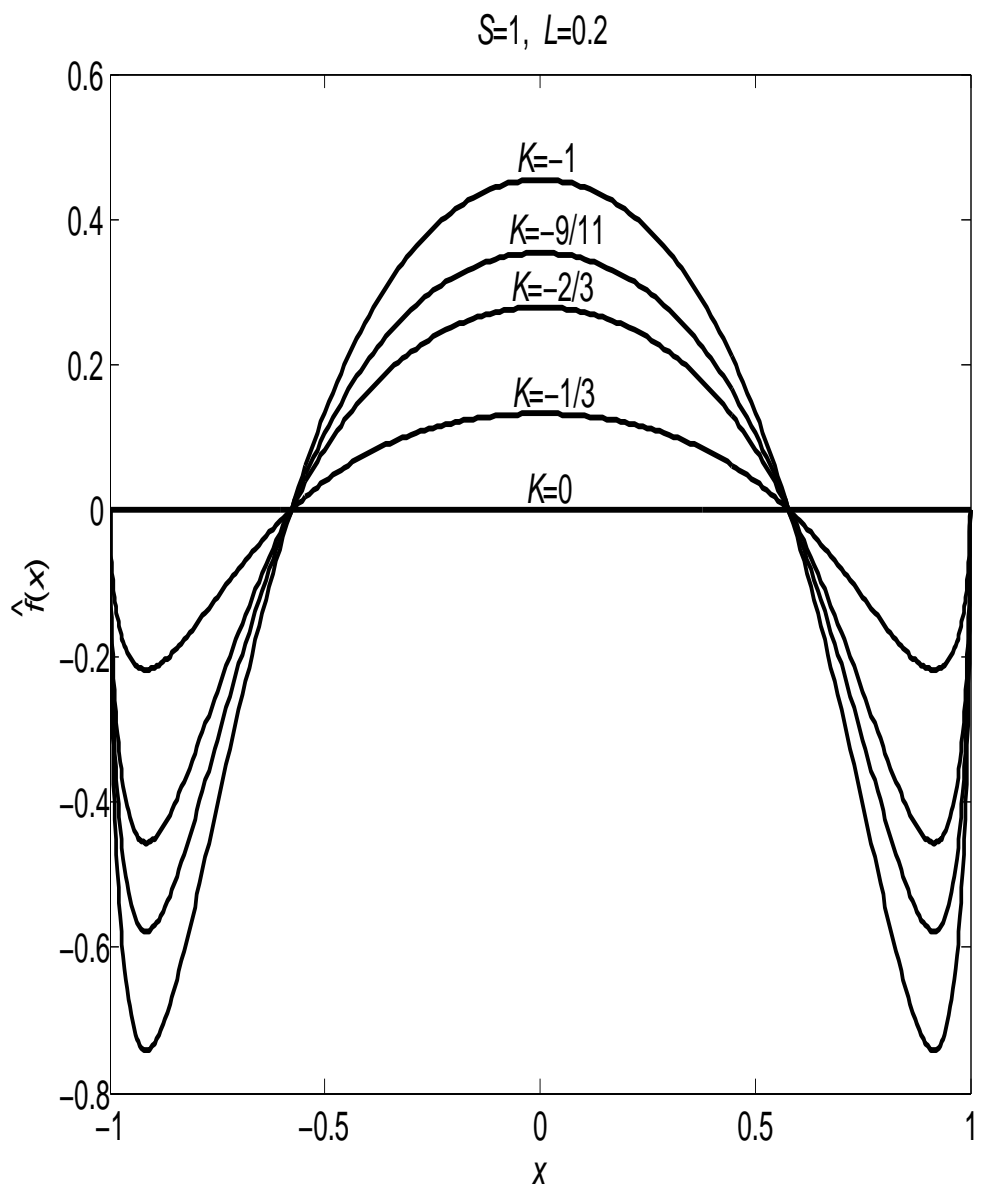

FiguRE 8. Variation of $\widehat{f}(x)$ for five different values of $K=$ $0,-1 / 3,-2 / 3,-9 / 11,-1$ with $S=1$ and $L=0.2$.

(i) $\max \left\{\sigma_{32}^{(1)} / \sigma_{32}^{\infty}\right\}=1.6245$ occurs at $x= \pm 0.922$ on the upper crack face very close the crack tips, $\min \left\{\sigma_{32}^{(1)} / \sigma_{32}^{\infty}\right\}=0.5552$ occurs at the center of the upper crack face;

(ii) $\max \left\{\sigma_{32}^{(2)} / \sigma_{32}^{\infty}\right\}=1.0893$ occurs just at the crack tips $x= \pm 1$, $\min \left\{\sigma_{32}^{(2)} / \sigma_{32}^{\infty}\right\}=0.909$ occurs at the center of the lower crack face; 


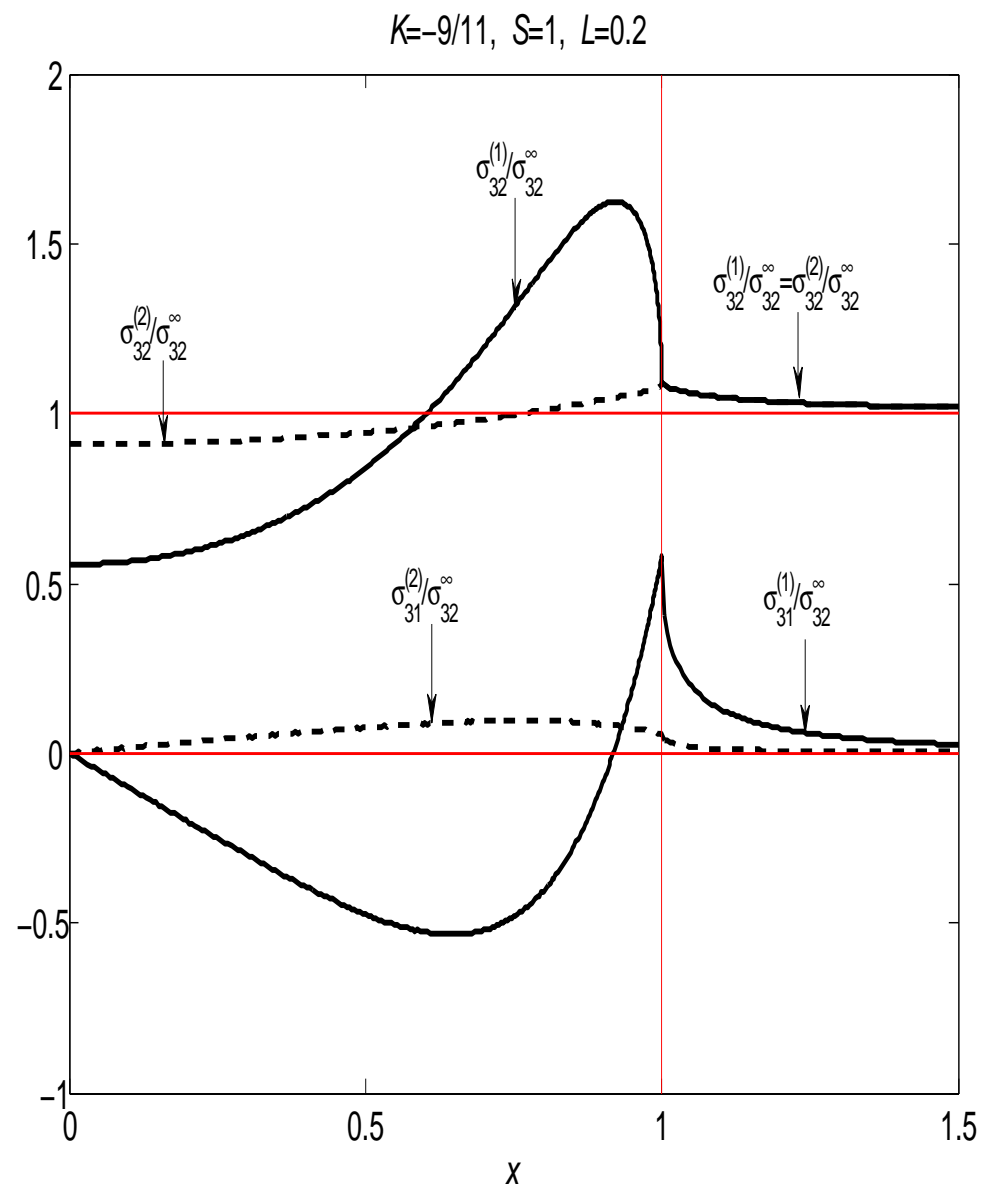

Figure 9. Continuous distributions of the stress components $\sigma_{32}^{(1)}, \sigma_{32}^{(2)}$, $\sigma_{31}^{(1)}$ and $\sigma_{31}^{(2)}$ along the bimaterial interface with $K=-9 / 11, S=1$ and $L=0.2$.

(iii) $\max \left\{\sigma_{31}^{(1)} / \sigma_{32}^{\infty}\right\}=0.5780$ occurs just at the right crack tip $x=1$ and at $x=-0.76$ on the upper crack face, $\min \left\{\sigma_{31}^{(1)} / \sigma_{32}^{\infty}\right\}=$ -0.5780 occurs at $x=0.65$ on the upper crack face and at the left crack tip $x=-1$; 
(iv) $\max \left\{\sigma_{31}^{(2)} / \sigma_{32}^{\infty}\right\}=0.0959$ occurs at $x=0.76$ on the lower crack face, $\min \left\{\sigma_{31}^{(2)} / \sigma_{32}^{\infty}\right\}=-0.0959$ occurs at $x=0.76$ on the lower crack face. In this example, the interface crack with surface strain gradient effects will induce a stress concentration factor $(\mathrm{SCF})$ of 1.6245. Our numerical results also show that an increase in $S$ and/or $L$ will suppress the variations of the stress components along the interface.

Figure 10 illustrates the induced SCF as a function of $S$ and $L$ with $K=-9 / 11$. It is observed from the figure that SCF is a monotonic decreasing function of both $S$ and $L$. Our result also shows that SCF is an increasing function of $|K|(-1<K<1)$ for fixed values of $S$ and $L$. This fact implies that the material mismatch of the two half-planes will enlarge the SCF.

Finally, we remark that our numerical solution demonstrates fast and accurate convergence. Specifically, the results in Figures 2-10 with $N=300$ are extremely close to the corresponding ones obtained by taking different values of $N<300$. This is illustrated in Table 1 where we list the values of $\{\max \hat{b}(x)\}, \max \{\hat{f}(x)\}, \min \{\hat{f}(x)\}, \max \{\Delta \widehat{w}\}$ in the case when $K=-9 / 11, S=1$ and $L=0.2$ for various values of $N$.

TABLE 1. The values of $\max \{\hat{b}(x)\}, \max \{\hat{f}(x)\}, \min \{\hat{f}(x)\}, \max \{\Delta \widehat{w}\}$ with $K=-9 / 11, S=1$ and $L=0.2$ for various values of $N$.

\begin{tabular}{|c|c|c|c|c|c|c|}
\hline$N$ & 10 & 20 & 40 & 60 & 100 & 300 \\
\hline $\max \{\hat{b}(x)\}$ & 0.2727 & 0.2657 & 0.2654 & 0.2653 & 0.2652 & 0.2652 \\
$\max \{\hat{f}(x)\}$ & 0.3540 & 0.3537 & 0.3538 & 0.3539 & 0.3539 & 0.3539 \\
$\min \{\hat{f}(x)\}$ & -0.5770 & -0.5777 & -0.5780 & -0.5781 & -0.5781 & -0.5781 \\
$\max \{\Delta \widehat{w}\}$ & 0.1676 & 0.1653 & 0.1649 & 0.1648 & 0.1647 & 0.1647 \\
\hline
\end{tabular}

7. Conclusions. In this study, we have incorporated the effects of surface strain gradient elasticity into the mode III interface crack problem arising in the anti-plane shear deformations of an isotropic bimaterial. Green's function method is used to obtain a system of coupled hyper-singular integro-differential equations which is solved numerically by means of Chebyshev polynomials and a collocation method. The complete semi-analytic solution obtained demonstrates that all the stress components remain finite at the crack tips. In the absence of the strain gradient effects presented here (i.e., when only the traditional model of surface elasticity is present), the stresses 


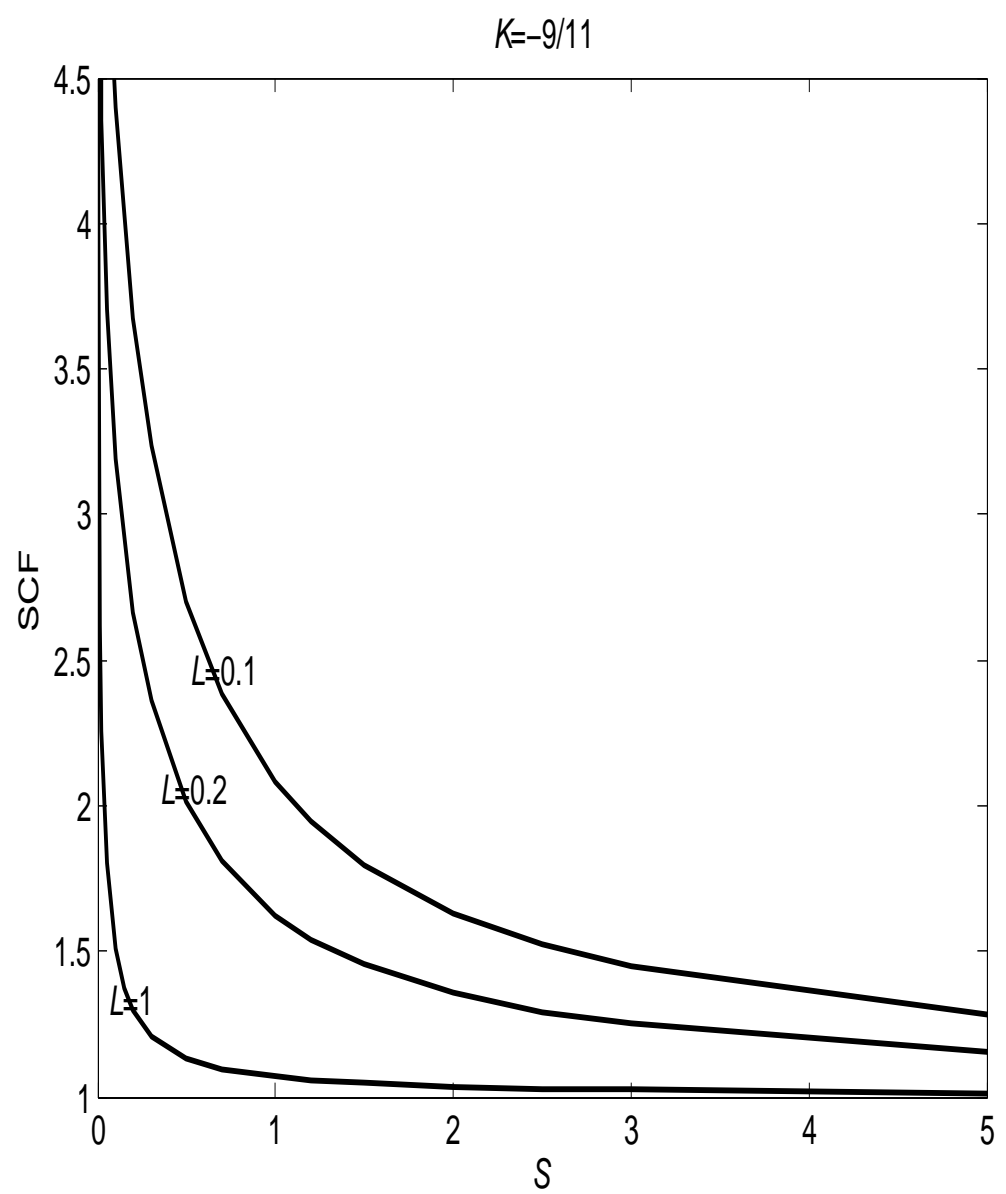

FigurE 10. The SCF as a function of $S$ and $L$ with $K=-9 / 11$.

again exhibit both the logarithmic and the square root singularities at the crack tips.

Acknowledgments. The authors are indebted to the editor and a referee for helpful comments and suggestions. 


\section{REFERENCES}

1. Y.A. Antipov and P. Schiavone, Integro-differential equation for a finite crack in a strip with surface effects, Q.J. Mech. Appl. Math. 64 (2011), 87-106.

2. Y.S. Chan, A.C. Fannjiang and G.H. Paulino, Integral equations with hypersingular kernelstheory and applications to fracture mechanics, Int. J. Eng. Sci. 41 (2003), 683-720.

3. A.C. Fannjiang, G.H. Paulino and Y.S. Chan, Strain gradient elasticity for antiplane shear cracks: a hypersingular integrodifferential equation approach, SIAM J. Appl. Math. 62 (2002), 1066-1091.

4. M.E. Gurtin and A. Murdoch, A continuum theory of elastic material surfaces, Arch. Rat. Mech. Anal. 57 (1975), 291-323.

5. M.E. Gurtin and A.I. Murdoch, Surface stress in solids, Int. J. Sol. Struct. 14 (1978), 431-440.

6. M.E. Gurtin, J. Weissmuller and F. Larche, A general theory of curved deformable interface in solids at equilibrium, Philos. Mag. 78 (1998), 1093-1109.

7. C.I. Kim, C.Q. Ru and P. Schiavone, A clarification of the role of cracktip conditions in linear elasticity with surface effects, Math. Mech. Sol. 18 (2013), $59-66$.

8. C.I. Kim, P. Schiavone and C.Q. Ru, The effects of surface elasticity on an elastic solid with mode-III crack: Complete solution, ASME J. Appl. Mech. 77 (2010), 021011-1-021011-7.

9. __ Analysis of a mode III crack in the presence of surface elasticity and a prescribed non-uniform surface traction, Z. Ang. Math. Phys. 61, 555-564.

10. Analysis of plane-strain crack problems (mode I and mode II) in the presence of surface elasticity, J. Elasticity 104 (2011), 397-420.

11. Mech. 63 (2011), 267-286.

12. Effect of surface elasticity on an interface crack in plane deformations, Proc. Roy. Soc. Lond. 467 (2011), 3530-3549.

13. X. Markenscoff and J. Dundurs, Annular inhomogeneities with eigenstrain and interphase modeling, J. Mech. Phys. Sol. 64 (2014), 468-482.

14. P.A. Martin, End-point behavior of solutions to hypersingular integral equations, Proc. Roy. Soc. Lond. 432 (1991), 301-320.

15. G.H. Paulino, A.C. Fannjiang and Y.S. Chan, Gradient elasticity theory for mode III fracture in functionally grade materials-Part I: Crack perpendicular to gradation, ASME J. Appl. Mech. 70 (2003), 531-542.

16. C.Q. Ru, Simple geometrical explanation of Gurtin-Murdoch model of surface elasticity with clarification of its related versions, Sci. China 53 (2010), 536544 .

17. D.J. Steigmann and R.W. Ogden, Plane deformations of elastic solids with intrinsic boundary elasticity, Proc. Roy. Soc. Lond. 453 (1997), 853-877. 
18. D.J. Steigmann and R.W. Ogden, Elastic surface-substrate interactions, Proc. Roy. Soc. Lond. 455 (1999), 437-474.

19. T.C.T. Ting, Anisotropic elasticity-theory and applications, Oxford University Press, New York, 1996.

20. I. Vardoulakis, G. Exadaktylos and E. Aifantis, Gradient elasticity with surface energy: Mode-III crack problem, Int. J. Sol. Struct. 33 (1996), 4531-4559.

21. J.R. Walton, A note on fracture models incorporating surface elasticity, J. Elasticity 109 (2012), 95-102.

22. X. Wang, A mode III arc shaped crack with surface elasticity, Z. Angew. Math. Phys. 66 (2015), 1987-2000.

23. X. Wang and H. Fan, Interaction between a nanocrack with surface elasticity and a screw dislocation, Math. Mech. Solids, in press.

24. X. Wang and P. Schiavone, Interaction between an edge dislocation and a crack with surface elasticity, ASME J. Appl. Mech. 82 (2015), 021006-1-021006-8.

25. X. Wang and K. Zhou, A mode III crack with surface strain gradient elasticity, 2014, unpublished.

26. _ A crack with surface effects in a piezoelectric material, Math. Mech. Solids, in press.

27. X. Wang, K. Zhou and M.S. Wu, Interface cracks with surface elasticity in anisotropic bimaterials, Int. J. Solids Struct. 59 (2015), 110-120.

School of Mechanical and Power Engineering, East China University of Science and Technology, 130 Meilong Road, Shanghai 200237, China

Email address: xuwang@ecust.edu.cn

Department of Mechanical Engineering, University of Alberta, 4-9 MeChanical Engineering Building Edmonton, Alberta Canada T6G 2G8

Email address: p.schiavone@ualberta.ca 